\title{
The reaction force: Three key points along an intrinsic reaction coordinate
}

\author{
PETER POLITZER, ${ }^{* a}$ ALEJANDRO TORO-LABBÉ, ${ }^{*},{ }^{\mathrm{b}}$ SOLEDAD GUTIÉRREZ-OLIVA, ${ }^{\mathrm{b}}$ \\ BÁRBARA HERRERA, ${ }^{\mathrm{b}}$ PABLO JAQUE, ${ }^{\mathrm{b}}$ MONICA C CONCHA ${ }^{\mathrm{a}}$ and JANE S MURRAY ${ }^{\mathrm{a}}$ \\ ${ }^{a}$ Department of Chemistry, University of New Orleans, New Orleans, LA 70148 USA \\ baboratorio de Química Teórica Computacional (QTC), Departamento de Química Física, Facultad de \\ Química, Pontificia Universidad Cátolica de Chile, Casilla 306, Correo 22, Santiago, Chile \\ e-mail: ato1a@pac.cl
}

\begin{abstract}
The concept of the reaction force is presented and discussed in detail. For typical processes with energy barriers, it has a universal form which defines three key points along an intrinsic reaction coordinate: the force minimum, zero and maximum. We suggest that the resulting four zones be interpreted as involving preparation of reactants in the first, transition to products in the second and third, and relaxation in the fourth. This general picture is supported by the distinctive patterns of the variations in relevant electronic properties. Two important points that are brought out by the reaction force are that (a) the traditional activation energy comprises two separate contributions, and (b) the transition state corresponds to a balance between the driving and the retarding forces.
\end{abstract}

Keywords. Reaction force; electronic properties; intrinsic reaction coordinate.

\section{The reaction force}

Any individual step in a chemical process normally involves some movements of atoms and changes in the structural parameters of the participants. The classical equations of motion can be used to establish the paths of minimum potential energy linking the transition state to the reactants and products; when expressed in mass-weighted Cartesian coordinates, this is the so-called "intrinsic reaction coordinate,", which we denote by $R_{c}$. It provides an effective and convenient basis for analyzing the course of the reaction step.

The variation of the potential energy $V$ of the system along $R_{c}$ typically resembles those shown in figures $1 \mathrm{a}$ and $2 \mathrm{a}$; the maximum, at $R_{c}=\beta$, corresponds to the transition state. The energy barriers between it and the reactants and products are the activation energies for the forward and reverse reactions, respectively; their relative heights vary from one reaction step to another. In 1955, Hammond postulated that the transition state is likely to resemble the reactants or the products, whichever are closer to it in potential energy. ${ }^{3}$

*For correspondence
The derivative of a potential energy with respect to position gives a force in the direction opposite to that of increasing energy. It is conceptually useful to apply this relationship to $V\left(R_{c}\right)$. $^{4-9}$

$$
\mathbf{F}\left(R_{c}\right)=-\partial V\left(R_{c}\right) / \partial R_{c} .
$$

We refer to $\mathbf{F}\left(R_{c}\right)$ as the "reaction force;" it is shown in figures $1 \mathrm{~b}$ and $2 \mathrm{~b}$. The form of $\mathbf{F}\left(R_{c}\right)$ is of course dictated by that of $V\left(R_{c}\right)$. As the latter initially increases rapidly, $\mathbf{F}\left(R_{c}\right)$ is negative and decreasing, until the first inflection point of $V\left(R_{c}\right)$ is reached, at $R_{c}=\alpha$. This is a minimum in $\mathbf{F}\left(R_{c}\right)$ :

$$
\left(\frac{\partial^{2} V\left(R_{c}\right)}{\partial R_{\mathrm{c}}^{2}}\right)_{\alpha}=0=\left(\frac{\partial \mathbf{F}\left(R_{c}\right)}{\partial R_{c}}\right)_{\alpha}
$$

After $R_{c}=\alpha, V\left(R_{c}\right)$ rises more slowly, and $\mathbf{F}\left(R_{c}\right)$ also begins to increase, but is still negative. At the transition state, $R_{c}=\beta$, which is the maximum of $V\left(R_{c}\right)$, $\mathbf{F}\left(R_{c}\right)$ reaches zero:

$$
\left(\frac{\partial V\left(R_{c}\right)}{\partial R_{c}}\right)_{\beta}=0=\mathbf{F}(\beta) .
$$


Following $R_{c}=\beta, V\left(R_{c}\right)$ starts its monotonic decrease, meaning that $\mathbf{F}\left(R_{c}\right)$ is henceforth positive. It reaches a maximum at the second inflection point $\gamma$ of $V\left(R_{c}\right)$,

$$
\left(\frac{\partial^{2} V\left(R_{c}\right)}{\partial R_{c}^{2}}\right)_{\gamma}=0=\left(\frac{\partial \mathbf{F}\left(R_{c}\right)}{\partial R_{c}}\right)_{\gamma},
$$

and then begins to diminish.

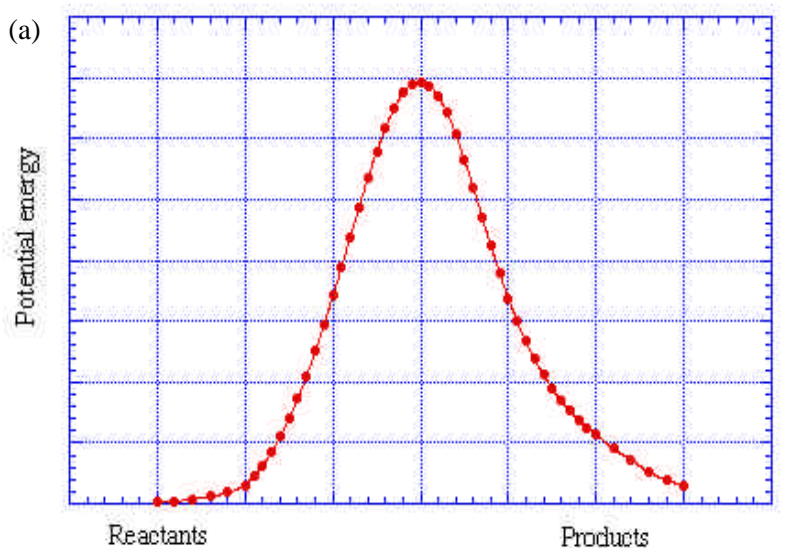

Intrinsic reaction coordinate

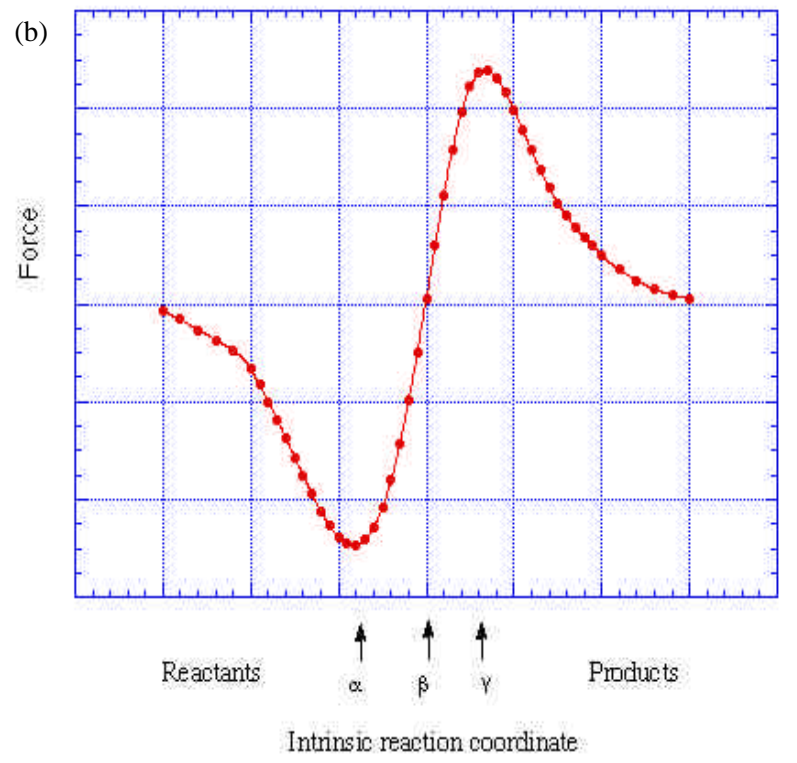

Figure 1. Potential energy $V\left(R_{c}\right),(\mathbf{a})$, and reaction force $\mathbf{F}\left(R_{c}\right),(\mathbf{b})$, along intrinsic reaction coordinate $R_{c}$. The positions of the reaction force minimum and maximum, $\alpha$ and $\gamma$, and the transition state, $\beta$, are indicated. The process is one for which the negative and the positive portions of $\mathbf{F}\left(R_{c}\right)$, taken separately, are approximately symmetric with respect to vertical lines through $R_{c}=\alpha$ and $R_{c}=\gamma$.
These general features of the reaction force are universal, independent of the specific chemical step as long as it involves an energy barrier such as those in figures 1a and 2a. (This precludes a simple bond rupture for which there is no barrier to the recombination of the fragments.) A key point that is brought out and emphasized by $\mathbf{F}\left(R_{c}\right)$ is that an intrinsic reaction coordinate is characterized by three welldefined points, $\alpha, \beta$ and $\gamma$. Thus the transition state is only one of three significant milestones along the reaction path, which can now be regarded as divided into four zones: Reactants $(\mathrm{R}) \rightarrow \alpha, \alpha \rightarrow \beta, \beta \rightarrow \gamma$

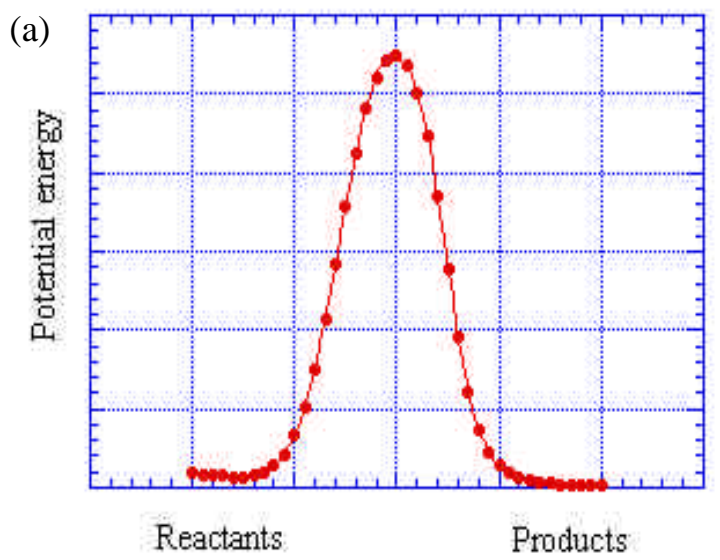

Intrinsic reaction coordinate

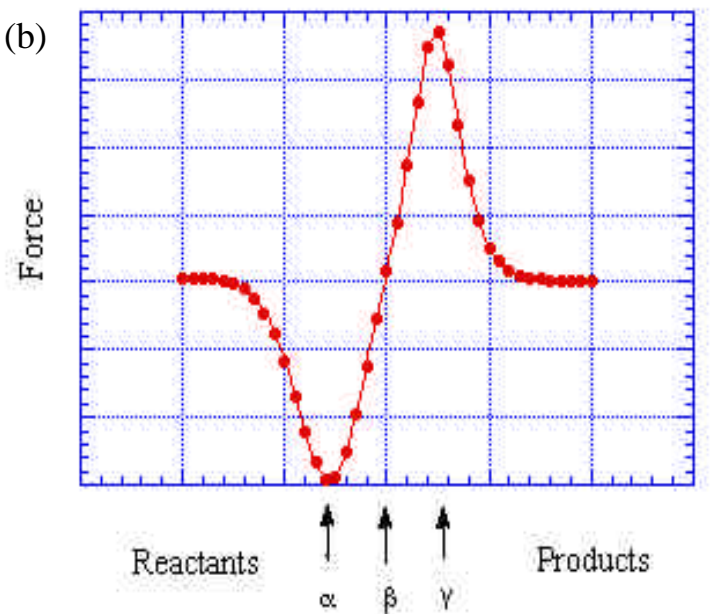

Intrinsic reaction coordinate

Figure 2. Potential energy $V\left(R_{c}\right),(\mathbf{a})$, and reaction force $\mathbf{F}\left(R_{c}\right)$, (b), along intrinsic reaction coordinate $R_{c}$. The positions of the reaction force minimum and maximum, $\alpha$ and $\gamma$, and the transition state, $\beta$, are indicated. The process is one for which the negative and the positive portions of $\mathbf{F}\left(R_{c}\right)$, taken separately, are asymmetric with respect to vertical lines through $R_{c}=\alpha$ and $R_{c}=\gamma$. 
and $\gamma \rightarrow$ products $(\mathrm{P})$. For the reverse reaction, $R_{c}$ increases in the opposite direction and the $\mathbf{F}\left(R_{c}\right)$ profile is the negative of that in figures $1 b$ or $2 b$.

For the overall process $\mathrm{R} \rightarrow \mathrm{P}$, the activation barrier $E_{\text {act }}$ and the total energy change $\Delta E_{\text {tot }}=E_{P}-E_{R}$ are related to $\mathbf{F}\left(R_{c}\right)$ by

$$
E_{\text {act }}=-\int_{\mathrm{R}}^{\beta} \mathbf{F}\left(R_{c}\right) \cdot \mathrm{d} R_{c}
$$

and

$$
\begin{aligned}
\Delta E_{\mathrm{tot}}=- & -\int_{\mathrm{R}}^{\mathrm{P}} \mathbf{F}\left(R_{c}\right) \cdot \mathrm{d} R_{c} \\
& \quad-\int_{\mathrm{R}}^{\beta} \mathbf{F}\left(R_{c}\right) \cdot \mathrm{d} R_{c}=-\int_{\beta}^{\mathrm{P}} \mathbf{F}\left(R_{c}\right) \cdot \mathrm{d} R_{c} .
\end{aligned}
$$

Thus $\Delta E_{\text {tot }}$ is equal to the difference in the areas under the positive and negative $\mathbf{F}\left(R_{c}\right)$ curves in figure $1 \mathrm{~b}$.

In general, $V(\mathrm{R}) \neq V(\mathrm{P})$; most reaction steps either release or absorb some energy. In contrast, $\mathbf{F}\left(R_{c}\right)$ is necessarily zero for both reactants and products, assuming that they are at equilibrium, i.e.,

$$
\mathbf{F}(\mathrm{R})=\mathbf{F}(\mathrm{P})=0 .
$$

In some instances, the positive and the negative portions of the $\mathbf{F}\left(R_{c}\right)$ curve, taken separately, are each relatively symmetric (figure 1). For example, the proton transfer process

$$
\mathrm{H}_{3} \mathrm{P}=\mathrm{O} \rightarrow \mathrm{H}_{2} \mathrm{P}-\mathrm{OH}
$$

is predicted computationally to have $\Delta E_{\text {tot }}=$ $-29.4 \mathrm{kcal} / \mathrm{mole}{ }^{8}$ However integrating $\mathbf{F}\left(R_{c}\right)$ over zones $\mathrm{R} \rightarrow \alpha$ and $\alpha \rightarrow \beta$ yields quite similar values, 26.2 and $25.1 \mathrm{kcal} / \mathrm{mole}$, respectively, as it does for zones $\beta \rightarrow \gamma$ and $\gamma \rightarrow \mathrm{P},-38.7$ and $-42.0 \mathrm{kcal} / \mathrm{mole}$. In such cases, there exist the approximate relationships,

$$
-\int_{\mathrm{R}}^{\alpha} \mathbf{F}\left(R_{c}\right) \cdot \mathrm{d} R_{c} \approx 0 \cdot 5 E_{\text {act }}
$$

and

$$
-\int_{\alpha}^{\gamma} \mathbf{F}\left(R_{c}\right) \cdot \mathrm{d} R_{c} \approx 0 \cdot 5 \Delta E_{\mathrm{tot}} .
$$

Martínez and Toro-Labbé have shown that (9) and (10) are satisfied by a group of nine one-step processes. $^{8}$
More common, however, is that the positive and negative segments of $\mathbf{F}\left(R_{c}\right)$ are each distinctly asymmetric. ${ }^{5-7,9}$ An example is in figure 2. Equations (9) and (10) are then not valid. This situation will be further analyzed in a later section.

\section{The four reaction zones}

What happens in the four zones defined by the points $\alpha, \beta$ and $\gamma$ on the intrinsic reaction coordinate? From figures $1 b$ and $2 b$, we infer the following sequence: The reaction is initially characterized by an increasing retarding force, which reaches its extremum at $R_{c}=\alpha$. At this point, a countering driving force begins to manifest itself. The overall effect is still retarding, but diminishingly so. At $R_{c}=\beta$, the driving and retarding forces are exactly equal; $\mathbf{F}\left(R_{c}\right)=0$. In this sense, therefore, the transition state is one of equilibrium, despite being associated with maximum energetic instability. After $R_{c}=\beta$, the driving force is dominant, and continues to become stronger until $R_{c}=\gamma$. It then begins to weaken and reaches zero for the product.

To what do the retarding and driving forces correspond, in structural and electronic terms? The details of this depend of course upon the specific process. For the ones that we have studied so far, ${ }^{4-9}$ which have been primarily intra- and intermolecular atom transfers, certain generalizations can be made.

The first zone, $R_{c}<\alpha$, involves preparation, e.g. rotations, conformational changes, etc., which will facilitate subsequent reaction, perhaps by bringing the interacting sites into closer proximity or by optimizing their relative configurations. The transition to products, as by bond breaking and formation, begins to be significant at $R_{c}=\alpha$; the former action would give rise to the retarding force, which would gradually diminish, the latter to the increasing driving force. The transition proceeds through the zones $\alpha \rightarrow \beta$ and $\beta \rightarrow \gamma$. After $R_{c}=\gamma$, the system relaxes to the final equilibrium geometry of the product or products.

This sequence of events is illustrated in figures 3 and 4 for the amine $\rightarrow$ imine tautomerization of adenine, (11), and the keto $\rightarrow$ enol of thymine, (12): ${ }^{7}$<smiles>Cc1ncnc2[nH]cnc12</smiles> 
<smiles>Cc1c[nH]c(O)nc1=O</smiles>

In the zone $R \rightarrow \alpha$, a key feature of each process is seen to be the bending motion of the transferring hydrogen toward the acceptor atom; the angle $\mathrm{H}-$ $\mathrm{N}$ (donor) $-\mathrm{C}$ decreases from $118^{\circ}$ to $87^{\circ}$ for adenine

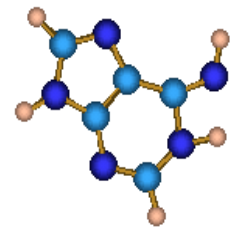

ForceMaximum

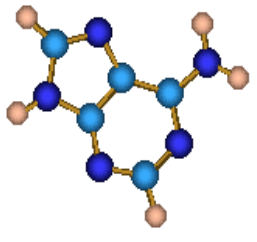

Rectat

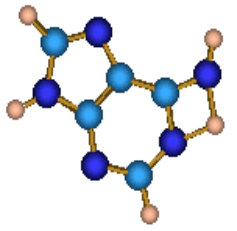

Transitionstde

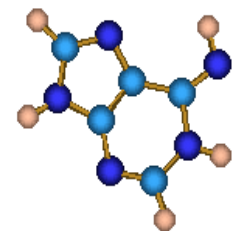

Produt

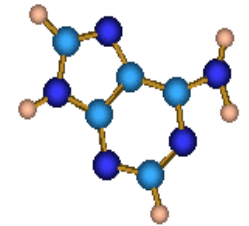

ForceMrimum
Figure 3. Structure of system at key points along intrinsic reaction coordinate of the amine $\rightarrow$ imine tautomerization of adenine (11).

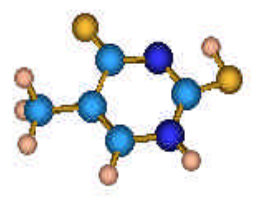

ForcelMaxmum

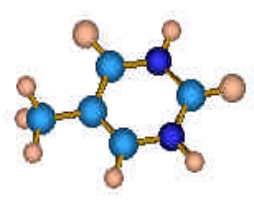

Readat

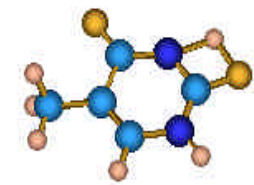

Trasitionste

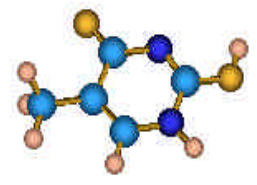

Produt

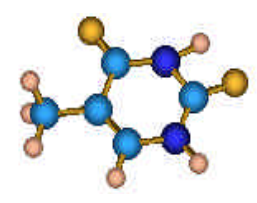

ForceMinimum
Figure 4. Structure of system at key points along intrinsic reaction coordinate of the keto $\rightarrow$ enol tautomerization of thymine (12). and from $116^{\circ}$ to $81^{\circ}$ for thymine (HF/6-311G**). The $\mathrm{N}$ (donor) $-\mathrm{H}$ distances do increase somewhat, by 0.12 and $0.14 \mathrm{~A}$, but much more between $R_{c}=\alpha$ and $R_{c}=\gamma, 0.54 \mathrm{~A}$ (adenine) and $0.37 \mathrm{~A}$ (thymine). At $R_{c}=\gamma$, the hydrogens are within 0.08 and $0.17 \mathrm{~A}$ of their final equilibrium distances from the acceptor atoms. The last zone again emphasizes bending, the $\mathrm{H}-\mathrm{N}$ (acceptor) $-\mathrm{C}$ angle of adenine and the $\mathrm{H}-\mathrm{O}$ (acceptor)-C of thymine both increasing by $26^{\circ}$.

The general picture that has been presented is supported by the variations in key electronic properties along the intrinsic reaction coordinates. In proton transfers, the donor atom's electrostatic potential is roughly constant and weakly negative in the zone $R \rightarrow \alpha$, in which primarily geometric rather than electronic changes are occurring. ${ }^{6}$ Between $\alpha$ and $\gamma$, however, the donor very rapidly becomes much more negative, as the proton moves away from it. After $R_{c}=\gamma$, the donor's potential varies little from its now quite negative value, as the system's structure relaxes to that of the product. The electrostatic potential of the acceptor shows exactly the reverse behavior.

The more negative is a given atom in a molecule, the lower is its local ionization energy. ${ }^{6}$ Thus the latter is initially high for the donor and remains so until $R_{c}=\alpha$; it then falls sharply between $R_{c}=\alpha$ and $R_{c}=\gamma$, whereupon it levels off. For the acceptor, we again see the opposite. This is shown in figure 5 for the keto $\rightarrow$ enol conversion of thymine, ${ }^{7}(12)$.

Analogous trends are observed for the electronic populations of bonds that are weakened (strengthened) during a process. ${ }^{5,7,9}$ They decrease (increase) mainly between $\alpha$ and $\gamma$, and are approximately constant in the $\mathrm{R} \rightarrow \alpha$ and $\gamma \rightarrow \mathrm{P}$ zones, where geometrical rearrangements are taking place.

The characteristic patterns seen in figure 5, resembling titration curves in analytical chemistry, have been found as well for other electronic properties - atomic charges, Fukui functions and dipole moments. ${ }^{5-9}$ They change slowly and gradually in the preparation and relaxation zones, $\mathrm{R} \rightarrow \alpha$ and $\gamma \rightarrow P$, which emphasize structural effects, but very rapidly in the transition zones, $\alpha \rightarrow \beta$ and $\beta \rightarrow \gamma$.

\section{The activation barrier, $E_{\text {act }}$}

From figures 1 and 2, it follows that the quantity traditionally denoted as the activation energy, $E_{\text {act }}$, can be viewed as the result of two contributions. The preceding discussion has suggested that these are as- 
sociated with the initial preparation and the first phase of the transition to products. The same applies to the reverse reaction.

In some cases, the two terms that comprise $E_{\text {act }}$ are quite similar, and (9) is valid. When this is not so, as in the process represented by figure 2 , our experience thus far has been that the energy needed for the preparation is likely to be the larger of the two. (The relative magnitudes can be determined qualitatively simply by comparing the areas under the $\mathbf{F}\left(\mathbf{R}_{\mathrm{c}}\right)$ curve from $\mathrm{R}$ to $\alpha$ and from $\alpha$ to $\beta$.) For example, the amine $\rightarrow$ imine and keto $\rightarrow$ enol tautomeriza-

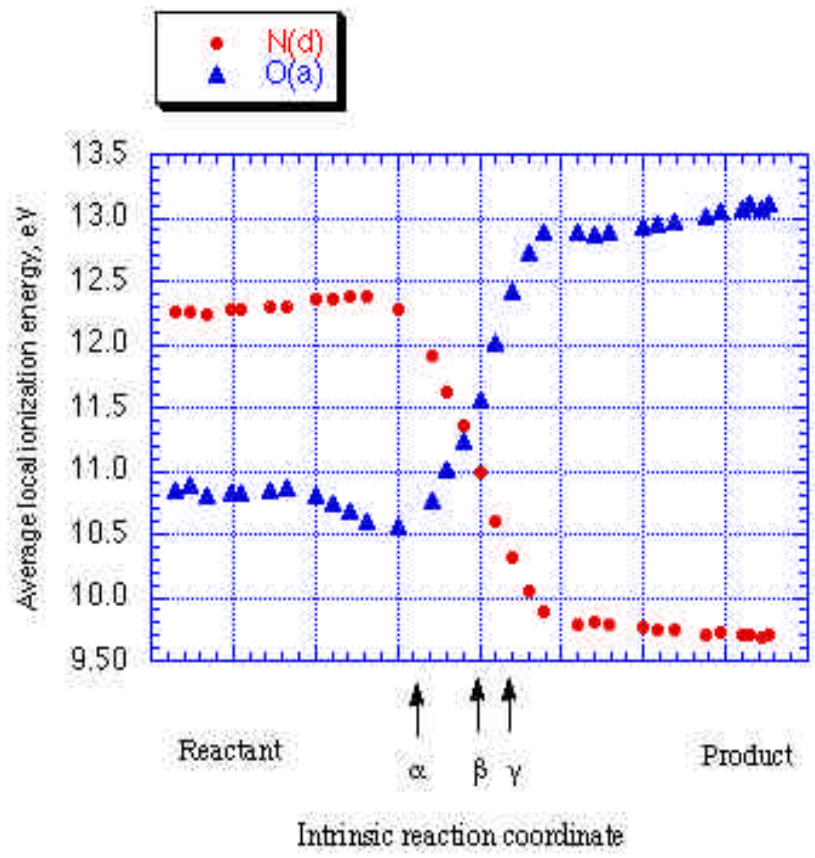

Figure 5. Variation of average local ionization energies of donor nitrogen and acceptor oxygen along intrinsic reaction coordinate in keto $\rightarrow$ enol tautomerization of thymine (12). The positions of the reaction force minimum and maximum, $\alpha$ and $\gamma$, and the transition state, $\beta$, are indicated.

Table 1. Computed energies (B3LYP/6-311G**) associated with (13) and (14), in kcal/mole [ref. 9].

\begin{tabular}{lcc}
\hline Energy & Equation (13) & Equation (14) \\
\hline$E_{\text {act }}($ forward) & 32.75 & 33.48 \\
$\Delta E(\mathrm{R} \rightarrow \alpha)$ & 22.35 & 22.72 \\
$\Delta E(\alpha \rightarrow \beta)$ & 10.40 & 10.76 \\
$E_{\text {act }}($ reverse $)$ & 31.32 & 26.49 \\
$\Delta E(\mathrm{P} \rightarrow \gamma)$ & 19.27 & 13.82 \\
$\Delta E(\gamma \rightarrow \beta)$ & 12.05 & 12.67 \\
\hline
\end{tabular}

tions of the four DNA bases require, at the MP2/6$311 \mathrm{G}^{* *}$ level, $35-39 \mathrm{kcal} / \mathrm{mole}$ in the $\mathrm{R} \rightarrow \alpha$ zone compared to $10-13 \mathrm{kcal} / \mathrm{mole}$ in the $\alpha \rightarrow \beta .^{7}$

Recognition that there are two contributions to $E_{\text {act }}$, which is a direct consequence of the $\mathbf{F}\left(R_{c}\right)$ profile, can provide some useful insights and clarify apparent anomalies. For example, since oxygen is a better hydrogen bond acceptor than is sulfur, it would be anticipated that the forward reactions in (13) and (14) below,<smiles>O=CCCCCCCCCCC(=O)SS</smiles>

will be promoted by the $\mathrm{O}-----\mathrm{H}$ interactions in the reactants more than are the reverse reactions by S-----H in the products. Yet the computed activation barriers $E_{\text {act }}$ are greater for the forward processes (table 1). ${ }^{9}$ This can be explained by decomposing each $E_{\text {act }}$ into its two components. Table 1 shows that, in each case, the reason that $E_{\text {act }}$ (forward) $>E_{\text {act }}$ (reverse) is because of the requirements of the preparation steps, $\mathrm{R} \rightarrow \alpha$ and $\mathrm{P} \rightarrow \gamma$. The portion of $E_{\text {act }}$ that relates to the actual hydrogen transfer is, as expected, less for the forward reaction, $\Delta E(\alpha \rightarrow \beta)$, than for the reverse, $\Delta E(\gamma \rightarrow \beta)$. Furthermore, the total energy associated with the two transition zones is negative in the forward direction, $\Delta E(\alpha \rightarrow \beta)+\Delta E(\beta \rightarrow \gamma)$, and positive in the reverse, $\Delta E(\gamma \rightarrow \beta)+\Delta E(\beta \rightarrow \alpha)$.

\section{Summary}

Our primary purpose in this paper is to draw attention to the fact that there are three key points associated with the course of any one-step process having an energy barrier such as shown in figures 1a and 2a. This is universal. Our interpretation of the four reaction zones - preparation in the first, transition to products (bond-breaking, -forming etc.) mainly in the second and third, relaxation in the fourth - may need to be modified as more processes are investigated. However, the key concepts will remain:

(a) the existence of four reaction zones, defined by the three key points $\alpha, \beta$ and $\gamma$; 
(b) the transition state as one of balance between the driving and retarding forces; and

(c) the activation barriers $E_{\text {act }}$ being composed of two distinct contributions.

These points follow not from interpretation but from the shapes of the $V\left(R_{c}\right)$ and $\mathbf{F}\left(R_{c}\right)$ profiles.

\section{Acknowledgements}

We appreciate the computational assistance of Pat Lane. This work was supported by FONDECYT 1020534. PJ is grateful to MECESUP (PUC-004, Red Química UCH-0116) for a postdoctoral fellowship. SGO and BH are grateful to the Project Programa Bicentenario en Ciencias y Tecnologia.

\section{References}

1. Fukui K 1981 Acc. Chem. Res. 14363

2. Gonzalez C and Schlegel H B 1990 J. Phys. Chem. 94 5523

3. Hammond G S 1955 J. Am. Chem. Soc. 77334

4. Toro-Labbé A 1999 J. Phys. Chem. A103 4398

5. Jaque P and Toro-Labbé A 2000 J. Phys. Chem. A104 995

6. Toro-Labbé A, Gutiérrez-Oliva S, Concha M C, Murray J S and Politzer P 2004 J. Chem. Phys. 121 4570

7. Herrera B and Toro-Labbé A 2004 J. Chem. Phys. 121 7096

8. Martínez J and Toro-Labbé A 2004 Chem. Phys. Lett. 392132

9. Gutiérrez-Oliva S, Herrera B, Toro-Labbé A and Chermette H 2005 J. Phys. Chem. A109 1748 